\title{
Spectrum dan Spectrum Laplacian pada Graf Mahkota
}

\author{
Khoirul Umam \\ Matematika, Institut Teknologi dan Bisnis Kalbis \\ Jalan Palomas Selatan kav.22, Jakarta Timur 13210 \\ Email: thoirul.umam@ikalbis.ac.id
}

\begin{abstract}
Chown Crown ( $S_{i v}^{0}$ is a graph that has the number of vertices $2 n$ and the number of edges are $n(n-1)$ with $n \geq 3, n \in$ integers. Suppose that $\lambda_{1}, \lambda_{2}, \ldots, \lambda_{n}$ are eigen values of $a$ matrix and $m\left(\lambda_{1}\right), m\left(\lambda_{2}\right), \ldots, m\left(\lambda_{n}\right)$ are the multiplicity of each $\lambda$, so the spectrum of a graph can be expressed as a matrix $2 \times n$ whose line elements are $\lambda_{1}, \lambda_{2}, \ldots, \lambda_{n}$ in the first row, and $\boldsymbol{m}\left(\lambda_{1}\right), \boldsymbol{m}\left(\lambda_{2}\right), \ldots, \boldsymbol{m}\left(\lambda_{n}\right)$ in the second row. In this paper, we will discuss spectrums from adjacency matrices and laplacian matrix spectrums of $S_{n}^{0}$.
\end{abstract}

Ieywords: crown graph, eigen value, multiplicity, spectrums

\begin{abstract}
Abstrak: Graf Mahkota atau Crown graph ( $\left.\boldsymbol{S}_{\mathbf{n}}^{\mathbf{0}}\right)$ merupakan graf yang mempunyai jumlah simpulnya $2 n$ dan jumlah busuonya $\boldsymbol{n}(\boldsymbol{n}-\mathbf{1})$ dengan $\boldsymbol{n} \geq \mathbf{3}, \boldsymbol{n} \boldsymbol{\epsilon}$ bilangan bulat. Misalkan $\lambda_{1}, \lambda_{2}, \ldots, \lambda_{n}$ adalah nilai-nilai eigen dari suatu matriks dam $m\left(\lambda_{1}\right), m\left(\lambda_{2}\right), \ldots, m\left(\lambda_{n}\right)$ adalah multiplisitas dari masing-masing $\lambda$, maka spectnum suatu graf dapat dinyatakan dengan matriks berordo $2 \times n$ yang elemen barismya $\lambda_{1}, \lambda_{2}, \ldots, \lambda_{n}$ pada baris pertama, dam $\boldsymbol{m}\left(\lambda_{1}\right), \boldsymbol{m}\left(\lambda_{2}\right), \ldots, \boldsymbol{m}\left(\lambda_{n}\right)$ pada baris kedua. Pada penelitian ini akan dibahas spectruon dari matriks adjacency dan spectrum matriks laplacian dari $S_{n}^{0}$.
\end{abstract}

Sata kunci: crown graph, graf mahkota, multiplisitas, nilai eigen, spectrums

\section{PENDAHULUAN}

Graf Mahkota atau sering disebut Crown graph dinotasikan dengan $S_{n}^{0}$. Graf Mahkota didefinisikan sebagai graf dengan himpunan simpul $\left\{x_{0}, x_{1}, \ldots, x_{n-1}, y_{0}, y_{1}, \ldots, y_{n-1}\right\}$ dan himpunan busur $\left\{\left(x_{i}, y_{i}\right): 0 \leq i, j \leq n-1, i \neq j\right\} \quad$ dengan $n \geq$ $3, n \in$ bilangan bulat. Graf mahkota juga dapat didefinisikan sebagai graf bipartit komplit $\left(K_{n, n}\right)$ dengan menghilangkan simpul horisontal [1].

Dari definisi tersebut diketahui bahwa jumlah simpul pada $S_{n}^{0}$ adalah $2 n$ dan jumlah busumya adalah $n(n-1)$ dengan $n \geq 3, n \in$ bilangan bulat. Sehingga dapat diperoleh jumlah simpul $S_{n}^{0}$ untuk $n=3,4,5,6, \ldots, n$ adalah $6,8,10,12, \ldots, 2 n$ Sedangkan jumlah busumya adalah $6,12,20,30$, $\ldots, n(n-1)$ [2]. Beberapa Graf Mahkota dapat dilihat pada Gambar 1.

Terdapat kasus khusus dari $S_{n}^{0}$ yaitu untuk $S_{3}^{0}$ dengan 6 simpul membentuk sebuah cycle, sedangkan untuk $S_{4}^{0}$ dengan 8 simpul merupakan isomorfis dari graf cube [2].
Misalkan $\lambda_{1}, \lambda_{2}, \ldots, \lambda_{n}$ adalah nilai-nilai eigen dari suatu matriks dan $m\left(\lambda_{1}\right), m\left(\lambda_{2}\right), \ldots, m\left(\lambda_{n}\right)$ adalah multiplisitas dari masing-masing $\lambda$, maka spectrum suatu graf $\mathrm{G}$ dapat didefinisikan sebagai himpunan nilai-nilai eigen yang dituliskan dengan multiplisitasnya[3]. Spectrum dinyatakan dengan matriks berordo $2 \times n$ yang elemen barisnya $\lambda_{1}, \lambda_{2}, \ldots, \lambda_{n}$ pada baris pertama, dan $m\left(\lambda_{1}\right), m\left(\lambda_{2}\right), \ldots, m\left(\lambda_{n}\right)$ pada baris kedua. Jika urutan nilai $\lambda_{1}>\lambda_{2}>\cdots>\lambda_{n}$ maka spektrum suatu matriks dapat dituliskan:

$$
\operatorname{Spec} A(G)=\left(\begin{array}{cccc}
\lambda_{1} & \lambda_{2} & \ldots & \lambda_{n} \\
m\left(\lambda_{1}\right) & m\left(\lambda_{2}\right) & \ldots & m\left(\lambda_{n}\right)
\end{array}\right)
$$

\section{METODE PENELITIAN}

Metode penelitian yang digunakan dalam penelitian ini adalah studi literatur. Penelitian ini bertujuan untuk menguraikan bentuk umum spectrum dan spectrum laplacian pada Graf Mahkota $\left(S_{n}^{0}\right)$. 
Makalah dan buku yang berkaitan dengan spectrum dan spectrum laplacian dipelajari, selanjutnya hasil studi literatur tersebut digunakan sebagai landasan teori untuk mendapatkan bukti formal untuk menemukan bentuk umum spectrum dan spectrum laplacian pada Graf Mahkota $\left(S_{n}^{0}\right)$.
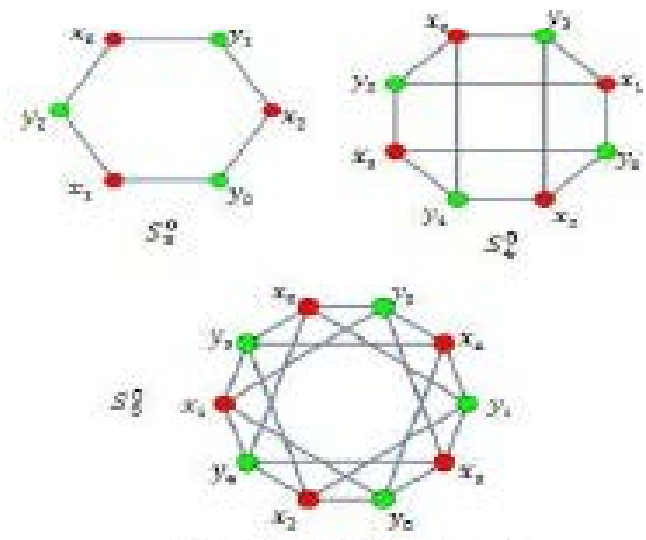

Gambar 1. Graf Mahrota

\section{HASIL DAN PEMBAHASAN}

Pembahasan bentuk umum spectrum dan spectrum laplacian pada Graf Mahkota $\left(S_{n}^{0}\right)$ akan diuraikan dalam bentuk teorema dan disertai buktinya. Namun Sebelumnya akan dilihat pola dari spectrum dan spectrum laplacian pada Graf Mahkota $\left(S_{n}^{0}\right)$.

A. Menemukan Pola Spectrum dan Spectrum Laplacian Pada Graf Mahkota $\left(S_{n}^{0}\right)$.

\section{Graf Mahkota $S_{3}^{0}$}

Graf Mahkota $S_{3}^{0}$ dapat dilihat pada Gambar 2.
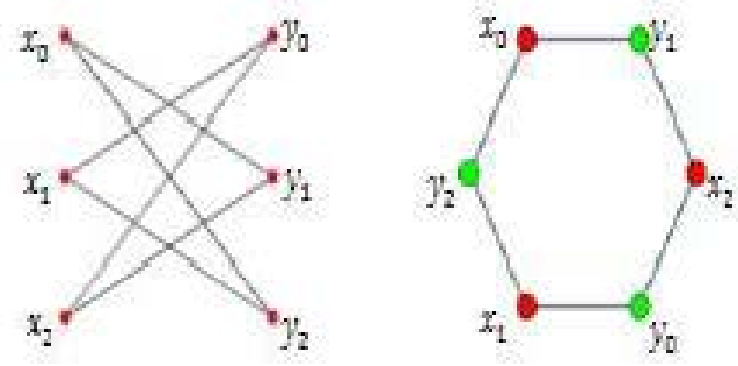

Gambar 2. Graf Mahkota $S_{5}^{\circ}$

Diperoleh matriks ketetanggaan dari $S_{3}^{0}$ :
$A\left(S_{3}^{0}\right)=\left(\begin{array}{llllll}0 & 0 & 0 & 0 & 1 & 1 \\ 0 & 0 & 0 & 1 & 0 & 1 \\ 0 & 0 & 0 & 1 & 1 & 0 \\ 0 & 1 & 1 & 0 & 0 & 0 \\ 1 & 0 & 1 & 0 & 0 & 0 \\ 1 & 1 & 0 & 0 & 0 & 0\end{array}\right)$

persamaan polynomial karakteristik $S_{3}^{0}$ :

$\phi\left(S_{3}^{0}\right)=\lambda^{6}-6 \lambda^{4}+9 \lambda^{2}-4$

$$
=(\lambda+2)(\lambda+1)^{2}(\lambda-1)^{2}(\lambda-2)
$$

$\operatorname{spec} A\left(S_{3}^{0}\right)=\left(\begin{array}{cccc}-2 & -1 & 1 & 2 \\ 1 & 2 & 2 & 1\end{array}\right)$

Diperoleh matriks laplacian dari $S_{3}^{0}$ :

$L\left(S_{3}^{0}\right)=\left(\begin{array}{cccccc}2 & 0 & 0 & 0 & -1 & -1 \\ 0 & 2 & 0 & -1 & 0 & -1 \\ 0 & 0 & 2 & -1 & -1 & 0 \\ 0 & -1 & -1 & 2 & 0 & 0 \\ -1 & 0 & -1 & 0 & 2 & 0 \\ -1 & -1 & 0 & 0 & 0 & 2\end{array}\right)$

persamaan polynomial karakteristik laplacian $S_{3}^{0}$ :

$\phi\left(s_{3}^{0}\right)=\lambda^{6}-12 \lambda^{5}+54 \lambda^{4}-112 \lambda^{3}+105 \lambda^{2}-36 \lambda$

$=\lambda(\lambda-1)^{2}(\lambda-3)^{2}(\lambda-4)$

$\operatorname{spec} L\left(S_{3}^{0}\right)=\left(\begin{array}{llll}0 & 1 & 3 & 4 \\ 1 & 2 & 2 & 1\end{array}\right)$

\section{Graf Mahkota $S_{4}^{0}$}

Graf Mahkota $S_{4}^{0}$ dapat dilihat pada Gambar 3.
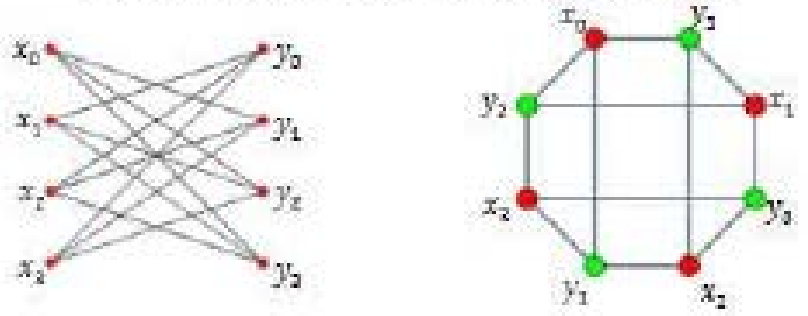

Gambar 3. Grof Mahbota $S_{4}^{0}$

Diperoleh matriks ketetanggaan dari $S_{4}^{0}$ :

$$
A\left(S_{4}^{0}\right)=\left(\begin{array}{llllllll}
0 & 0 & 0 & 0 & 0 & 1 & 1 & 1 \\
0 & 0 & 0 & 0 & 1 & 0 & 1 & 1 \\
0 & 0 & 0 & 0 & 1 & 1 & 0 & 1 \\
0 & 0 & 0 & 0 & 1 & 1 & 1 & 0 \\
0 & 1 & 1 & 1 & 0 & 0 & 0 & 0 \\
1 & 0 & 1 & 1 & 0 & 0 & 0 & 0 \\
1 & 1 & 0 & 1 & 0 & 0 & 0 & 0 \\
1 & 1 & 1 & 0 & 0 & 0 & 0 & 0
\end{array}\right)
$$

diperoleh persamaan polynomial karakteristik $S_{4}^{0}$ :

$\phi\left(C_{4}\right)=\lambda^{8}-12 \lambda^{6}+30 \lambda^{4}-28 \lambda^{2}+9$

$$
=(\lambda+3)(\lambda+1)^{3}(\lambda-1)^{3}(\lambda-3)
$$


$\operatorname{spec} A\left(S_{4}^{0}\right)=\left(\begin{array}{cccc}-3 & -1 & 1 & 3 \\ 1 & 3 & 3 & 1\end{array}\right)$

Diperoleh matriks laplacian dari $S_{4}^{0}$ :

$$
\begin{aligned}
& L\left(S_{4}^{0}\right) \\
& =\left(\begin{array}{cccccccc}
3 & 0 & 0 & 0 & 0 & -1 & -1 & -1 \\
0 & 3 & 0 & 0 & -1 & 0 & -1 & -1 \\
0 & 0 & 3 & 0 & -1 & -1 & 0 & -1 \\
0 & 0 & 0 & 3 & -1 & -1 & -1 & 0 \\
0 & -1 & -1 & -1 & 3 & 0 & 0 & 0 \\
-1 & 0 & -1 & -1 & 0 & 3 & 0 & 0 \\
-1 & -1 & 0 & -1 & 0 & 0 & 3 & 0 \\
-1 & -1 & -1 & 0 & 0 & 0 & 0 & 3
\end{array}\right)
\end{aligned}
$$

diperoleh persamaan polynomial karakteristik laplacian $S_{4}^{0}$ :

$$
\begin{aligned}
\phi\left(S_{4}^{0}\right)= & \lambda^{8}-24 \lambda^{7}+240 \lambda^{6}-1296 \lambda^{5}+4080 \lambda^{4} \\
& -7488 \lambda^{3}+7424 \lambda^{2}-3072 \lambda \\
= & \lambda(\lambda-2)^{3}(\lambda-4)^{3}(\lambda-6)
\end{aligned}
$$$$
\operatorname{spec} L\left(S_{4}^{0}\right)=\left(\begin{array}{llll}
0 & 2 & 4 & 6 \\
1 & 3 & 3 & 1
\end{array}\right)
$$

\section{Graf Mahkota $S_{5}^{0}$}

Graf Mahkota $S_{\varsigma}^{0}$ dapat dilihat pada Gambar 4.
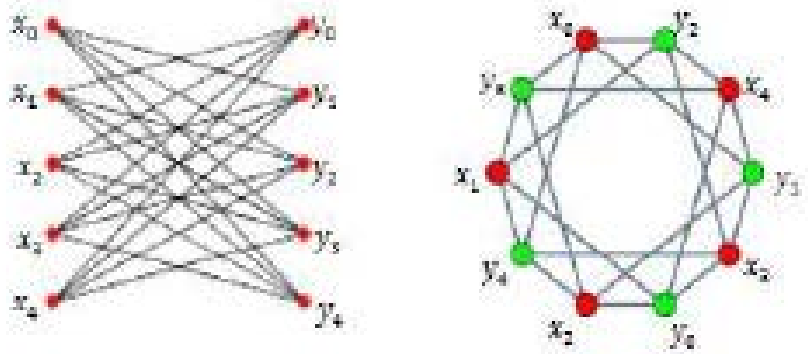

Gumbar 4. Graff Mahbota $S_{5}^{0}$

Diperoleh matriks ketetanggaan dari $S_{5}^{0}$ :

$$
A\left(S_{5}^{0}\right)=\left(\begin{array}{llllllllll}
0 & 0 & 0 & 0 & 0 & 0 & 1 & 1 & 1 & 1 \\
0 & 0 & 0 & 0 & 0 & 1 & 0 & 1 & 1 & 1 \\
0 & 0 & 0 & 0 & 0 & 1 & 1 & 0 & 1 & 1 \\
0 & 0 & 0 & 0 & 0 & 1 & 1 & 1 & 0 & 1 \\
0 & 0 & 0 & 0 & 0 & 1 & 1 & 1 & 1 & 0 \\
0 & 1 & 1 & 1 & 1 & 0 & 0 & 0 & 0 & 0 \\
1 & 0 & 1 & 1 & 1 & 0 & 0 & 0 & 0 & 0 \\
1 & 1 & 0 & 1 & 1 & 0 & 0 & 0 & 0 & 0 \\
1 & 1 & 1 & 0 & 1 & 0 & 0 & 0 & 0 & 0 \\
1 & 1 & 1 & 1 & 0 & 0 & 0 & 0 & 0 & 0
\end{array}\right)
$$

diperoleh persamaan polynomial karakteristik $S_{5}^{0}$ :

$$
\begin{gathered}
\phi\left(S_{5}^{0}\right)=\lambda^{10}-20 \lambda^{8}+70 \lambda^{6}-100 \lambda^{4}+65 \lambda^{2}-16 \\
=\lambda(\lambda+3)^{4}(\lambda-5)^{4}(\lambda-8) \\
\text { spec } A\left(S_{5}^{0}\right)=\left(\begin{array}{cccc}
-4 & -1 & 1 & 4 \\
1 & 4 & 4 & 1
\end{array}\right)
\end{gathered}
$$

Diperoleh matriks laplacian dari $S_{5}^{0}$ :

$L\left(s_{5}^{0}\right)=$

$\left(\begin{array}{cccccccccc}4 & 0 & 0 & 0 & 0 & 0 & -1 & -1 & -1 & -1 \\ 0 & 4 & 0 & 0 & 0 & -1 & 0 & -1 & -1 & -1 \\ 0 & 0 & 4 & 0 & 0 & -1 & -1 & 0 & -1 & -1 \\ 0 & 0 & 0 & 4 & 0 & -1 & -1 & -1 & 0 & -1 \\ 0 & 0 & 0 & 0 & 4 & -1 & -1 & -1 & -1 & 0 \\ 0 & -1 & -1 & -1 & -1 & 4 & 0 & 0 & 0 & 0 \\ -1 & 0 & -1 & -1 & -1 & 0 & 4 & 0 & 0 & 0 \\ -1 & -1 & 0 & -1 & -1 & 0 & 0 & 4 & 0 & 0 \\ -1 & -1 & -1 & 0 & -1 & 0 & 0 & 0 & 4 & 0 \\ -1 & -1 & -1 & -1 & 0 & 0 & 0 & 0 & 0 & 4\end{array}\right)$ diperoleh persamaan polynomial karakteristik laplacian $S_{5}^{0}$ :

$$
\begin{aligned}
& \phi\left(S_{5}^{0}\right)=\lambda^{10}- 40 \lambda^{9}+700 \lambda^{8}-7040 \lambda^{7} \\
&+44870 \lambda^{6}-188048 \lambda^{5} \\
&+518460 \lambda^{4}-907200 \lambda^{3} \\
&+914625 \lambda^{2}-405000 \lambda \\
&=\lambda(\lambda-3)^{4}(\lambda-5)^{4}(\lambda-8)
\end{aligned}
$$

\begin{tabular}{|c|c|}
\hline Graf Mahkota & Spectrum \\
\hline$S_{3}^{0}$ & $\left(\begin{array}{cccc}-2 & -1 & 1 & 2 \\
1 & 2 & 2 & 1\end{array}\right)$ \\
\hline$S_{4}^{0}$ & $\left(\begin{array}{cccc}-3 & -1 & 1 & 3 \\
1 & 3 & 3 & 1\end{array}\right)$ \\
\hline$S_{5}^{0}$ & $\left(\begin{array}{cccc}-4 & -1 & 1 & 4 \\
1 & 4 & 4 & 1\end{array}\right)$ \\
\hline .. & ... \\
\hline$S_{n}^{0}$ & $\left(\begin{array}{cccc}1-n & -1 & 1 & n \\
1 & n-1 & n-1\end{array}\right.$ \\
\hline
\end{tabular}

$\operatorname{spec} L\left(S_{4}^{0}\right)=\left(\begin{array}{llll}0 & 3 & 5 & 8 \\ 1 & 4 & 4 & 1\end{array}\right)$

Dari perhitungan spectrum dan spectrum laplacian dari Graf Mahkota diatas diperoleh Tabel 1 dan Tabel 2. Pada Tabel 1 diperoleh bentuk umum untuk spectrum Graf Mahkota $\left(S_{n}^{0}\right)$ yaitu

$$
\operatorname{Spec} A\left(S_{n}^{0}\right)=\left(\begin{array}{cccc}
1-n & -1 & 1 & n-1 \\
1 & n-1 & n-1 & 1
\end{array}\right) .
$$

Sedangkan pada Tabel 2 diperoleh bentuk umum untuk spectrum laplacian dari Graf Mahkota $\left(S_{n}^{0}\right)$ yaitu

$$
\operatorname{Spec} L\left(S_{n}^{0}\right)=\left(\begin{array}{cccc}
0 & n-2 & n & 2 n-2 \\
1 & n-1 & n-1 & 1
\end{array}\right) .
$$

Tabel I Spectrum dari Graf Mahkota 
Tabel 2 Spectrum laplacian dari Graf Mahbota

\begin{tabular}{|c|c|}
\hline Graf Mahkota & Spectrum Laplacian \\
\hline$S_{3}^{0}$ & $\left(\begin{array}{llll}0 & 1 & 3 & 4 \\
1 & 2 & 2 & 1\end{array}\right)$ \\
\hline$S_{4}^{0}$ & $\left(\begin{array}{llll}0 & 2 & 4 & 6 \\
1 & 3 & 3 & 1\end{array}\right)$ \\
\hline$S_{5}^{0}$ & $\left(\begin{array}{llll}0 & 3 & 5 & 8 \\
1 & 4 & 4 & 1\end{array}\right)$ \\
\hline$\ldots$ & $\ldots$ \\
\hline$S_{n}^{0}$ & $\left(\begin{array}{cccc}0 & n-2 & n & 2 n-2 \\
1 & n-1 & n-1 & 1\end{array}\right)$ \\
\hline
\end{tabular}

B. Teorema dan Bukti Formal dari Spectrum dan Spectrum Laplacian pada Graf Mahkota $\left(S_{\mathrm{n}}^{0}\right)$.

Teorema 1. Misalkan $S_{n}^{0}$ adalah Graf Mahkota, maka maka spectrum dari $S_{n}^{0}$ adalah

$$
\operatorname{Spec} A\left(S_{n}^{0}\right)=\left(\begin{array}{cccc}
1-n & -1 & 1 & n-1 \\
1 & n-1 & n-1 & 1
\end{array}\right)
$$

\section{Bukti Formal}

Misalkan $A\left(S_{n}^{0}\right)$ adalah matriks adjacency dari Graf Mahkota [4], maka

$$
\begin{aligned}
A\left(S_{n}^{0}\right) & =\left(\begin{array}{cccc:cccc}
0 & 0 & \cdots & 0 & 0 & 1 & \cdots & 1 \\
0 & 0 & \cdots & 0 & 1 & 0 & \cdots & 1 \\
\vdots & \vdots & \ddots & \vdots & \vdots & \vdots & \ddots & \vdots \\
0 & 0 & \cdots & 0 & 1 & 1 & \cdots & 0 \\
\hdashline 0 & 1 & \cdots & 1 & 0 & 0 & \cdots & 0 \\
1 & 0 & \cdots & 1 & 0 & 0 & \cdots & 0 \\
\vdots & \vdots & \ddots & \vdots & \vdots & \vdots & \ddots & \vdots \\
1 & 1 & \cdots & 0 & 0 & 0 & \cdots & 0
\end{array}\right) \\
& =\left(\begin{array}{ccc}
0 & A\left(K_{n}\right) \\
\hdashline A\left(K_{n}\right) & 0 & 0
\end{array}\right)
\end{aligned}
$$

$A\left(S_{n}^{0}\right)$ dapat dipartisi menjadi matriks 0 dan $A\left(K_{n}\right)$, dengan $K_{n}$ adalah graf reguler. Karena elemen $A\left(S_{n}^{0}\right)$ disusun oleh $A\left(K_{n}\right)$ maka nilai-nilai eigen $A\left(S_{n}^{0}\right)$ dapat dibentuk dari $A\left(K_{n}\right)$ [5]. Jones (2013) telah menjelaskan bahwa septrum $A\left(K_{n}\right)$ adalah $\operatorname{Spec} A\left(K_{n}\right)=\left(\begin{array}{cc}-1 & n-1 \\ n-1 & 1\end{array}\right)$. Multiplisitas $A\left(S_{n}^{0}\right)$ sama dengan dua kali multlipisitas $A\left(K_{n}\right)$, dikarenakan $A\left(S_{n}^{0}\right)$ disusun dari dua $A\left(K_{n}\right)$ [6].

Misalkan $\lambda$ adalah nilai-nilai eigen dari $A\left(K_{n}\right), \lambda^{*}$ adalah nilai-nilai eigen dari $A\left(S_{n}^{0}\right)$ dan $\mathbf{v}_{\lambda}$ adalah vektor eigen yang bersesuaian dengan $\lambda$, kemudian misalkan $\mathbf{x}=\left(\begin{array}{c}\mathbf{v}_{\lambda} \\ \mathbf{v}_{\lambda}\end{array}\right)$ dan $\mathbf{y}=\left(\begin{array}{c}\mathbf{v}_{\lambda} \\ -\mathbf{v}_{\lambda}\end{array}\right)$. Maka untuk $\mathbf{x}$ diperoleh:

$$
\begin{aligned}
A\left(S_{n}^{0}\right) \mathbf{x} & =\left(\begin{array}{cc}
\mathbf{0} & A\left(K_{n}\right) \\
A\left(K_{n}\right) & \mathbf{0}
\end{array}\right)\left(\begin{array}{l}
\mathbf{v}_{\lambda} \\
\mathbf{v}_{\lambda}
\end{array}\right) \\
& =\left(\begin{array}{l}
A\left(K_{n}\right) \cdot \mathbf{v}_{\lambda} \\
A\left(K_{n}\right) \cdot \mathbf{v}_{\lambda}
\end{array}\right) \\
& =\left(\begin{array}{l}
\lambda \cdot \mathbf{v}_{\lambda} \\
\lambda \cdot \mathbf{v}_{\lambda}
\end{array}\right) \\
& =\lambda \mathbf{x} \\
& =\lambda^{*} \mathbf{x}
\end{aligned}
$$

Jadi untuk $\mathbf{x}$ diperoleh $\lambda^{*}=\lambda$, maka

- untuk $\lambda=-1, \lambda^{*}=\lambda=-1$ dengan

multiplisitas $n-1$.

- untuk $\lambda=n-1, \lambda^{*}=\lambda=n-1$ dengan multiplisitas 1.

Kemudian untuk vertor $\mathbf{y}$, diperoleh

$$
\begin{aligned}
A\left(S_{n}^{0}\right) \mathbf{y} & =\left(\begin{array}{cc}
\mathbf{0} & A\left(K_{n}\right) \\
A\left(K_{n}\right) & \mathbf{0}
\end{array}\right)\left(\begin{array}{c}
\mathbf{v}_{\lambda} \\
-\mathbf{v}_{\lambda}
\end{array}\right) \\
& =\left(\begin{array}{c}
A\left(K_{n}\right) \cdot \mathbf{v}_{\lambda} \\
A\left(K_{n}\right) \cdot \mathbf{v}_{\lambda}
\end{array}\right) \\
& =\left(\begin{array}{c}
\lambda \cdot-\mathbf{v}_{\lambda} \\
\lambda \cdot \mathbf{v}_{\lambda}
\end{array}\right) \\
& =\left(\begin{array}{c}
-\lambda \cdot \mathbf{v}_{\lambda} \\
-\lambda \cdot-\mathbf{v}_{\lambda}
\end{array}\right) \\
& =-\lambda \mathbf{y} \\
& =\lambda^{*} \mathbf{y}
\end{aligned}
$$

Jadi untuk $\mathbf{y}$ diperoleh $\lambda^{*}=-\lambda$, maka

- untuk $\lambda=-1, \lambda^{*}=-\lambda=-(-1)=1$ dengan multiplisitas $n-1$.

- untuk $\lambda=n-1, \lambda^{*}=-\lambda=-(-(n-1))=$ $1-n$ dengan multiplisitas 1.

terbukti bahwa

$$
\operatorname{Spec} A\left(S_{n}^{0}\right)=\left(\begin{array}{cccc}
1-n & -1 & 1 & n-1 \\
1 & n-1 & n-1 & 1
\end{array}\right)
$$

Teorema 2. Misalkan $S_{n}^{0}$ adalah Graf Mahkota maka spectrum laplacian dari $S_{n}^{0}$ adalah

$$
\operatorname{Spec} L\left(S_{n}^{0}\right)=\left(\begin{array}{cccc}
0 & n-2 & n & 2 n-2 \\
1 & n-1 & n-1 & 1
\end{array}\right) \text {. }
$$

\section{Bukti Formal}

Misalkan $L\left(S_{n}^{0}\right)$ adalah matriks laplacian dari Graf Mahkkota [5], maka 


$$
\begin{aligned}
& L\left(S_{n}^{0}\right)= \\
& \left(\begin{array}{cccc|cccc}
(n-1) & 0 & \cdots & 0 & 0 & -1 & \cdots & -1 \\
0 & (n-1) & \cdots & 0 & -1 & 0 & \cdots & -1 \\
0 & 0 & \vdots & \vdots & -1 & -1 & \vdots & \vdots \\
0 & 0 & \cdots & (n-1) & -1 & -1 & \cdots & 0 \\
\hdashline 0 & -1 & \cdots & -1 & (n-1) & 0 & \cdots & 0 \\
-1 & 0 & \cdots & -1 & 0 & (n-1) & \cdots & 0 \\
-1 & -1 & \vdots & \vdots & 0 & 0 & \checkmark & \vdots \\
-1 & -1 & \cdots & 0 & 0 & 0 & \cdots & (n-1)
\end{array}\right) \\
& =\left(\begin{array}{lll}
(n-1) I & -K_{n} \\
\hdashline-K_{n} & (n-1) I
\end{array}\right)
\end{aligned}
$$

Dengan metode yang sama dengan pembuktian Teorema 1 , Misalkan $\lambda$ adalah nilai-nilai eigen dari $L\left(K_{n}\right), \lambda^{*}$ adalah nilai-nilai eigen dari $A\left(S_{n}^{0}\right)$ dan $\mathbf{v}_{\lambda}$ adalah vektor eigen yang bersesuaian dengan $\lambda$. kemudian misalkan

$$
\mathbf{x}=\left(\begin{array}{l}
\mathbf{v}_{\lambda} \\
\mathbf{v}_{\lambda}
\end{array}\right) \text { dan } \mathbf{y}=\left(\begin{array}{c}
\mathbf{v}_{\lambda} \\
-\mathbf{v}_{\lambda}
\end{array}\right)
$$

Maka diperoleh

$$
\begin{aligned}
L\left(S_{n}^{0}\right) \mathbf{x} & =\left(\begin{array}{ll}
(n-1) I & -A\left(K_{n}\right) \\
-A\left(K_{n}\right) & (n-1) I
\end{array}\right)\left(\begin{array}{l}
\mathbf{v}_{\lambda} \\
\mathbf{v}_{\lambda}
\end{array}\right) \\
& =\left(\begin{array}{c}
(n-1) \cdot \mathbf{v}_{\lambda}-A\left(K_{n}\right) \cdot \mathbf{v}_{\lambda} \\
-A\left(K_{n}\right) \cdot \mathbf{v}_{\lambda}+(n-1) \cdot \mathbf{v}_{\lambda}
\end{array}\right) \\
& =\left(\begin{array}{c}
(n-1) \cdot \mathbf{v}_{\lambda}-\lambda \cdot \mathbf{v}_{\lambda} \\
-\lambda \cdot \mathbf{v}_{\lambda}+(n-1) \cdot \mathbf{v}_{\lambda}
\end{array}\right) \\
& =\left(\begin{array}{c}
(n-1-\lambda) \cdot \mathbf{v}_{\lambda} \\
(n-1-\lambda) \cdot \mathbf{v}_{\lambda}
\end{array}\right) \\
& =(n-1-\lambda) \mathbf{x} \\
& =\lambda^{*} \mathbf{x}
\end{aligned}
$$

Jadi untuk $\mathbf{x}$ diperoleh $\lambda^{*}=(n-1-\lambda)$, maka

- untuk $\lambda=-1, \lambda^{*}=(n-1-\lambda)=-(n-1+$ $1=n$ dengan multiplisitas $n-1$.

- untuk $\lambda=n-1, \lambda^{*}=(n-1-\lambda)=$ $(n-1-(n-1))=0$ dengan multiplisitas 1 .

Kemudian untuk vertor $\mathbf{y}$, diperoleh

$$
\begin{aligned}
L\left(S_{n}^{0}\right) \mathbf{y}= & \left(\begin{array}{cc}
(n-1) I & -A\left(K_{n}\right) \\
-A\left(K_{n}\right) & (n-1) I
\end{array}\right)\left(\begin{array}{c}
\mathbf{v}_{\lambda} \\
-\mathbf{v}_{\lambda}
\end{array}\right) \\
& =\left(\begin{array}{c}
(n-1) \cdot \mathbf{v}_{\lambda}+A\left(K_{n}\right) \cdot \mathbf{v}_{\lambda} \\
-A\left(K_{n}\right) \cdot \mathbf{v}_{\lambda}-(n-1) \cdot \mathbf{v}_{\lambda}
\end{array}\right) \\
& =\left(\begin{array}{c}
(n-1) \cdot \mathbf{v}_{\lambda}+\lambda \cdot \mathbf{v}_{\lambda} \\
-\lambda \cdot \mathbf{v}_{\lambda}-(n-1) \cdot \mathbf{v}_{\lambda}
\end{array}\right) \\
& =\left(\begin{array}{c}
(n-1+\lambda) \cdot \mathbf{v}_{\lambda} \\
(n-1+\lambda) \cdot-\mathbf{v}_{\lambda}
\end{array}\right) \\
& =(n-1+\lambda) \mathbf{y} \\
& =\lambda^{*} \mathbf{y}
\end{aligned}
$$

Jadi untuk y diperoleh $\lambda^{*}=(n-1+\lambda)$. maka - untuk $\lambda=-1, \lambda^{*}=(n-1+\lambda)=(n-1-$ 1) $=n-2$ dengan multiplisitas $n-1$.
- untuk $\lambda=n-1, \lambda^{*}=(n-1-\lambda)=$ $(n-1+(n-1))=2 n-2$ dengan multiplisitas 1.

terbukti bahwa

$$
\text { Spec } L\left(S_{n}^{0}\right)=\left(\begin{array}{cccc}
0 & n-2 & n & 2 n-2 \\
1 & n-1 & n-1 & 1
\end{array}\right) .
$$

Bukti lain:

$$
\begin{aligned}
L\left(S_{n}^{0}\right) & =D\left(S_{n}^{0}\right)-A\left(G S_{n}^{0}\right) \\
& =k I-A(G)
\end{aligned}
$$

dengan $D\left(S_{n}^{0}\right)$ adalah matrik diagonal $S_{n}^{0}$ dan $k$ derajat setiap simpul di $S_{n}^{0}$ yaitu $k=n-1$

$$
\begin{aligned}
L\left(G_{n}\right) \mathbf{x} & =\left(k I-A\left(K_{n}\right)\right) \mathbf{x} \\
& =k \mathbf{x}-\lambda \mathbf{x} \\
& =(k-\lambda) \mathbf{x}
\end{aligned}
$$

Dimana $\lambda$ adalah nilai-nilai eigen dari $A\left(K_{n}\right)$ dan multiplisitas dari $(k-\lambda)$ sama dengan multiplisitas dari $\lambda$.

Sehingga didapat:

- untuk $\lambda=1-n$, maka $k-\lambda=(n-1)-$ $(1-n)=2 n-2($ multiplisitasnya 1$)$

- untuk $\lambda=-1$, maka $k-\lambda=(n-1)-$ $(-1)=n$ (multiplisitasnya $n-1$ )

- untuk $\lambda=1$, maka $k-\lambda=(n-1)-(1)=$ $n-2$ (multiplisitasnya $n-1$ )

- untuk $\lambda=n-1$, maka $k-\lambda=(n-1)-$ $(n-1)=0$ (multiplisitasnya 1)

terbukti bahwa

$\operatorname{Spec} L\left(S_{n}^{0}\right)=\left(\begin{array}{cccc}0 & n-2 & n & 2 n-2 \\ 1 & n-1 & n-1 & 1\end{array}\right)$.

\section{SIMPULAN}

Berdasarkan hasil pembahasan, dapat disimpulkan bahwa spectrum Graf Mahkota $\left(S_{n}^{0}\right)$ adalah

$$
\operatorname{Spec} A\left(S_{n}^{0}\right)=\left(\begin{array}{cccc}
1-n & -1 & 1 & n-1 \\
1 & n-1 & n-1 & 1
\end{array}\right)
$$

dan spectrum laplacian dari Graf Mahkota $\left(S_{n}^{0}\right)$ adalah

$$
\operatorname{Spec} L\left(S_{n}^{0}\right)=\left(\begin{array}{cccc}
0 & n-2 & n & 2 n-2 \\
1 & n-1 & n-1 & 1
\end{array}\right) .
$$

Penelitian ini masih dapat dikembangkan lagi, misalnya menentukan spectrum dan spectrum laplacian pada kelas graf lain misalnya pada graf shadow, graf fan, gaf koctail, graf matahari dan sebagainya. 


\section{DAFTAR RUJUKAN}

[1] M. Glen, S. Kitaev, and A. Pyatkin, "On the representation number of a crown graph," Discret. Appl. Math., vol. 244, pp. 89-93, Jul. 2018.

[2] S. Severini and E. W. Weisstein, "Crown Graph," p. 1, 2018.

[3] N. Biggs, "Algebraic Graph Theory." Cambridge University Press, New York, p. 7, 1993.
[4] K. A. Sugeng, S. Slamet, and D. Riama, Teori Graf dan Aplikasinya. Depok: Departemen Matematika FMIPA Universitas Indonesia, 2014, 36.

[5] R. B. Bapat, Graphs and Matrices. New Delhi: Springer, $2014,26,47$.

[6] O. Jones, "Spectra of Simple Graphs," pp. 1-20, 2013. 